\title{
Oromucosal Paste Dosage Form
}

National Cancer Institute

\section{Source}

National Cancer Institute. Oromucosal Paste Dosage Form. NCI Thesaurus. Code C149737.

Semi-solid single-dose or multidose preparation consisting of a paste of solid particles finely dispersed in a hydrophilic basis intended for oromucosal use. Oromucosal pastes are applied to the oral cavity or onto a specific part of the oral cavity, to obtain a local effect. 